\title{
AVALIAÇÃO DOS SINTOMAS E DA QUALIDADE DE VIDA DAS MULHERES NO CLIMATÉRIO
}

\author{
Anelise Silva RIBEIRO ${ }^{1}$ \\ Ana Karoliny Acerbi SOARES ${ }^{2}$ \\ Vanessa Martins de Souza SIQUEIRA ${ }^{3}$ \\ *Walnéia Aparecida de SOUZA ${ }^{4}$ \\ Márcia Helena Miranda Cardoso PODESTÁ ${ }^{4}$ \\ Eric Batista FERREIRA ${ }^{4}$
}

\author{
${ }^{1}$ Farmacêutica residente do curso de Residência Multiprofissional em Saúde da Família da Universidade Federal \\ de Alfenas-MG. \\ ${ }^{2}$ Acadêmica do Curso de Farmácia da Universidade Federal de Alfenas-MG. \\ ${ }^{3}$ Médica do PSF Santa Teresa de Guanhães -MG. \\ ${ }^{4}$ Professores da Universidade Federal de Alfenas-MG. \\ *Autor corresponde: walne@ unifal-mg.edu.br \\ Universidade Federal de Alfenas- UNIFAL-MG.
}

Recebido em: 07/11/2014 - Aprovado em: 05/06/2015 - Disponibilizado em: 15/07/2015

\section{Resumo}

Objetivo. Avaliar os sintomas do climatério e a influência destes sobre a qualidade de vida das mulheres a fim de que se possa direcionar-lhes uma assistência mais adequada e possibilitar a implantação de políticas de saúde que lhes confiram melhor qualidade de vida.

Métodos. Estudo descritivo, exploratório e comparativo realizado com 80 mulheres com idade entre 40 a 65 anos percentes às Unidades de Saúde do Município de Alfenas. Estas responderam o instrumento de Saúde da Mulher e foram avaliadas de acordo com o índice de Kupperman e Blatt .

Resultados. As mulheres apresentam em sua maioria sintomas leves. Não houve diferença no grau dos sintomas e na qualidade de vida de saúde da mulher nos estágios de perimenopausa e na pós-menopausa. Verificou-se que o índice de Kupperman e Blatt apresentou uma relacão com a qualidade de vida, sendo que quanto maior o índice de Kupperman e Blatt pior a qualidade de vida.

Conclusões. Neste estudo, a maioria das mulheres no climatério apresentaram sintomas leves e a qualidade de vida mostrou-se influencida pelos sintomas identificados. A presença dos sintomas leva as mulheres a procurar por unidades de saúde. Faz-se necessário que estas unidades estejam preparadas para um atendimento humazido e holístico a fim de que as mulheres sejam atendidas de forma integral e tenham suas necessidades sanadas, proporcionando, assim, uma melhora na qualidade de vida.

Palavras-chave: climatério; qualidade de vida; menopausa.

\section{EVALUATION OF SYMPTOMS AND QUALITY OF LIFE OF CLIMATERIC WOMEN}

\begin{abstract}
Objectives. Assess climacteric symptoms and their influence on the quality of life of women in order that it can direct to a more appropriate assistance and enable the implementation of health policies that give them a better quality of life.
\end{abstract}


Methods. A descriptive, exploratory and comparative study carried out with 80 women aged 40 to 65 from Health Units from the city of Alfenas. They answered the instrument of Women's Health and were evaluated according to the Kupperman and Blatt index.

Results. Women present, in most cases, mild symptoms. There was no difference in the degree of symptoms and quality of life of women's health in the stages of perimenopause and postmenopause. It was found that the rate of Kupperman and Blatt showed a relationship with the quality of life, being that the larger the Kupperman and Blatt index worse the quality of life.

Conclusions. In this study, the majority of climacteric women have mild symptoms and the quality of life was influenced by symptoms identified. The presence of symptoms lead women to look for health units. It is necessary that these units are prepared for a humane and holistic care to ensure that women are assisted in full and have their needs remedied thereby providing an improvement in the quality of life.

Keywords: climacteric; quality of life; menopause.

\section{INTRODUÇÃO}

A expectativa de vida da população mundial vem crescendo nas últimas décadas, o que possibilita que mais mulheres experienciem as transformações decorrentes do climatério (CARVALHO; GARCIA, 2003).

O climatério representa o período de transição da fase reprodutiva da vida da mulher para a senilidade. Ao longo desse extenso período de tempo, ocorrem modificações biopsicossociais que se instalam de maneira insidiosa e de forma variável, as quais configuram, em seu conjunto, a passagem do menacme para a senilidade (FERNANDES, 2003).

O climatério é dividido três fases: a fase pré-menopausal (do final do menacme ao momento da menopausa); a fase perimenopausal (período de 2 anos que precede e sucede a menopausa); e a fase pós-menopausal (iniciada 2 anos após a menopausa e finda na senectude) ( ASSOCIAÇÃO BRASILEIRA DE CLIMATÉRIO, 2004).

Estima-se que as mulheres permaneçam aproximadamente um terço de suas vidas em estado de deficiência hormonal (MORAES; VANDENBERGHE; SILVEIRA, 2007). O climatério é um período importante e inevitável na vida, devendo ser encarado como um processo natural e não como doença. Às vezes, é vivenciado como uma passagem silenciosa (sem queixas); outras vezes, essa fase pode ser muito expressiva, acompanhada de sintomatologia que gera alterações na rotina, mas, no geral, é uma fase com perdas e de ganhos, de altos e de baixos, de novas liberdades, de novas limitações e possibilidades para as mulheres (BRASIL, 2008).

Assim, na atenção à saúde durante o climatério, informações detalhadas precisam ser oferecidas, principalmente em relação às variadas facetas dessa nova etapa 
da vida, encorajando a mulher a vivê-la com mais energia, coragem e a aprender os limites e as oportunidades do processo de envelhecimento, abrangendo as transformações que ocorrem durante esse período (BRASIL, 2008).

A avaliação dos sintomas climatéricos pode ser realizada por meio do índice de Kupperman e Blatt, que consiste na avaliação de vários sintomas, medidos de acordo com a intensidade do acometimento referido pela paciente. Para cada sintoma, é estabelecido um peso diferente, de acordo com sua intensidade (KUPPERMAN; BLATT, 1953).

A relação entre esses sintomas e a qualidade de vida referente à saúde ainda é um assunto controverso e complexo, pois essa relação pode sofrer influências de valores religiosos, éticos e culturais (GALLON; WENDER, 2012). Mesmo assim, tem sido um tema frequente em pesquisas, porque seus resultados podem ajudar a definir condutas terapêuticas, assim como avaliar a relação custo/benefício do cuidado prestado (SILVA FILHO; COSTA, 2008).

Em 1992, Hunter desenvolveu o Women's Health Questionnaire (WHS) um instrumento para avaliar a saúde da mulher de meia-idade, que visa analisar mudanças físicas e no bem-estar decorrentes do período do climatério, bem como das alterações associadas à idade. Sua adaptação para a população brasileira (idioma e cultura) só ocorreu 10 anos mais tarde com a publicação da versão para o português do Questionário de Saúde da Mulher-QSM

(BONGANHA; MADRUGA, 2010).

É cada vez maior o número de mulheres que se preocupam em ter uma vida saudável, livre de incapacidades, de doenças e de sintomas desagradáveis que prejudicam o lazer, os relacionamentos interpessoais e o trabalho, mais do que apenas ter vida longa. As características de uma vida saudável são a essência do que significa qualidade de vida relacionada à saúde (SILVA FILHO; COSTA, 2008).

Assim, o estudo apresenta como objetivo avaliar os sintomas do climatério e a influência destes sobre a qualidade de vida das mulheres por meio do instrumento da saúde da mulher e o do índice de Kupperman e Blatt a fim de que se possa direcionar uma assistência mais adequada e possibilitar a implantação de políticas de saúde que lhes confiram melhor qualidade de vida. 


\section{METODOLOGIA}

Trata-se de um estudo descritivo, exploratório e comparativo, realizado com 80 mulheres com idade entre 40 a 65 anos, residentes na cidade de Alfenas, Minas Gerais, Brasil, entre agosto de 2013 e janeiro de 2014.

Foram escolhidas aleatoriamente, mulheres das Unidades de Atenção à Saúde e mulheres pertencentes ao município que apresentavam sintomas do climatério, mas que ainda não estavam na menopausa (perimenopausa) e mulheres na pósmenopausa, que concordaram em participar do estudo.

Após a assinatura do Termo de Consentimento Livre e Esclarecido, foram aplicados 3 instrumentos de coleta de dados. No primeiro, investigaram as características sociodemográfica, os hábitos de vida, as co-morbidades e a utilização de medicamentos. Em seguida, foram aplicados os instrumentos de avaliação dos sintomas do climatério (Índice Menopausal de Kupperman e Bratt) e o de qualidade de saúde da mulher.

A pesquisa foi aprovada pelo Comitê de Ética em Pesquisa da Universidade Federal de Alfenas - MG, conforme a resolução 196/96 do Conselho Nacional de Saúde para pesquisa envolvendo seres humanos, conforme protocolo $\mathrm{n}^{\mathrm{o}} 237.751$.

Para a avaliação dos sintomas do climatério, foi utilizado o Índice Menopausal de Kupperman e Bratt (IMKB). No IMBK, os diversos sintomas incluídos sob a denominação de síndrome climatérica (fogachos; insônia; parestesia; nervosismo; melancolia; vertigem; artralgia/mialgia; cefaleia; palpitação e zumbido) recebem valores numéricos de acordo com a sua intensidade (leves $=1$; moderados $=2 ; \quad$ severos $=3$ ) multiplicados pelos fatores de conversão de acordo com sua importância na síndrome climatérica. Dessa forma, os sintomas vasomotores podem receber valores 4,8 ou 12 ; a parestesia, a insônia e o nervosismo, 2, 4 ou 6; e o restante (melancolia; vertigem; artralgia/mialgia; cefaleia; palpitação e zumbido), os valores 1,2 ou 3 . De acordo com o somatório dos valores, os sintomas são classificados em leve (19), moderado (20-35) e severo (>35) (MARTINS et al., 2009).

A qualidade de vida foi analisada por meio do Instrumento Women's Health Questionnaire (WHQ) traduzido para o português. Esse instrumento avalia a Saúde da Mulher (QSM) e consta de 37 questões, oferecendo quatro alternativas como possibilidade de resposta. Suas questões 
estão divididas em sete grupos, dispostos aleatoriamente, que avaliam: depressão (sete questões) - 3;5;7;8;10;12;25; sintomas somáticos (sete questões)$14 ; 15 ; 16 ; 18 ; 23 ; 30 ; 35$;

memória/concentração (três questões) 20;33;36; sintomas vasomotores (duas questões) - 19;27; ansiedade/temores (quatro questões) - 2;4;6;9; comportamento sexual (três questões) - 24;31;34; problemas de sono ( três questões) $1 ; 11 ; 29$; sintomas menstruais (quatro questões) - 17;22;26;28; e atratividade (três questões) - 13;21;32 (DIAS et al., 2002)

As respostas obtidas no QSM foram convertidas em escores de forma que as pontuações 1 e 2 correspondem a 1 e as pontuações 3 e 4 correspondem a 0 . A pontuação total e a pontuação em cada domínio foram convertidas em porcentagem. Para a classificação da qualidade de vida, foram usados os tercis de forma que a mesma foi classificada em alta (0 a $27 \%$ ), média (27 a 46\%) e baixa (46 a 100\%).

Os dados dos questionários foram processados em banco de dados, por meio do programa Microsoft ${ }^{\circledR}$ Office Excel®, versão 2010, da Microsoft Corporation e para análise estatística, foi usado o $R$ Core Team (2013).
A avaliação do QSM foi realizada separadamente para cada tipo de sintoma. Foram aplicados os testes exatos de Fisher e o teste de qui-quadrado.

\section{RESULTADOS}

Das 80 mulheres entrevistadas, a idade média foi de 53,9 anos $( \pm 6,5)$, sendo $64 \%$ brancas; $53,8 \%$ apresentam mais de 5 anos de estudo e $73,8 \%$ possuem ocupação remunerada. Com relação à renda familiar, $45 \%$ recebem mais de 3 salários mínimos e $63,8 \%$ possuem um companheiro fixo. Os resultados estão apresentados na Tabela 1. 
Tabela 1: Distribuição das variáveis sociodemográficas das mulheres entrevistadas, Alfenas, 2013-2014.

\begin{tabular}{|c|c|c|}
\hline Variáveis & $\mathrm{n}$ & $\%$ \\
\hline \multicolumn{3}{|l|}{ Idade } \\
\hline $40-49$ & 21 & 26,3 \\
\hline $50-59$ & 40 & 50 \\
\hline $60-65$ & 19 & 23,8 \\
\hline \multicolumn{3}{|c|}{ Anos completos de estudo } \\
\hline$<5$ & 37 & 46,3 \\
\hline $5-9$ & 7 & 8,8 \\
\hline$\geq 10$ & 36 & 45 \\
\hline \multicolumn{3}{|l|}{ Cor } \\
\hline Branca & 64 & 80 \\
\hline Não branca & 16 & 20 \\
\hline \multicolumn{3}{|l|}{ Renda familiar } \\
\hline$<3$ & 44 & 55 \\
\hline $03-05$ & 16 & 20 \\
\hline$\geq 5$ & 20 & 25 \\
\hline \multicolumn{3}{|l|}{ Ocupação remunerada } \\
\hline Sim & 59 & 73,8 \\
\hline \multicolumn{3}{|l|}{ Estado marital } \\
\hline Com companheiro fixo & 51 & 63,8 \\
\hline Sem companheiro fixo & 29 & 36,3 \\
\hline
\end{tabular}

Das entrevistadas, $53,8 \%$ medicamentos estão apresentados na apresentam sobrepeso e/ou obesidade; 50\%, Tabela 2.

circunferência abdominal acima do normal;

e $50 \%$ não praticam atividade física regularmente.

Os dados em relação aos hábitos de vida, co-morbidades e uso de 
Tabela 2: Distribuição dos hábitos de vida, co-morbidades e uso de medicamentos das mulheres entrevistadas, Alfenas, 2013-2014.

\begin{tabular}{|c|c|c|}
\hline Variáveis & $\mathrm{n}$ & $\%$ \\
\hline \multicolumn{3}{|l|}{ Tabagismo } \\
\hline Sim & 12 & 15 \\
\hline Não & 68 & 85,01 \\
\hline \multicolumn{3}{|c|}{ Uso de bebidas alcoólicas } \\
\hline Sim & 13 & 16,25 \\
\hline Não & 67 & 83,75 \\
\hline \multicolumn{3}{|c|}{ Atividade física regular } \\
\hline Sim & 40 & 50 \\
\hline Não & 40 & 50 \\
\hline \multicolumn{3}{|l|}{ Reposição hormonal } \\
\hline Sim & 12 & 15 \\
\hline Não & 68 & 85 \\
\hline \multicolumn{3}{|l|}{ Co-morbidades } \\
\hline Sim & 60 & 75 \\
\hline Não & 20 & 25 \\
\hline \multicolumn{3}{|l|}{ Faz uso de medicação } \\
\hline Sim & 58 & 72,5 \\
\hline Não & 22 & 27,5 \\
\hline \multicolumn{3}{|l|}{ Nível de estresse } \\
\hline Sempre & 23 & 28,75 \\
\hline A maioria das vezes & 8 & 10 \\
\hline Algumas vezes & 26 & 32,5 \\
\hline Raramente & 23 & 28,75 \\
\hline
\end{tabular}

$\mathrm{Na}$ análise dos medicamentos utilizados, hipocolesterolêmico e $8,8 \%$, de $40 \%$ fazem uso de anti-hipertensivos; antidiabéticos orais. $22,5 \%$ de ansiolíticos e antidepressivos; $16,3 \%$ de hormônio tireoidiano; $12,5 \%$ de 
No estudo do IMKB, verificou-se que os sintomas leves foram em maior proporção nas mulheres no climatério quando comparados com outros sintomas. $\mathrm{Na}$ comparação do grau dos sintomas das mulheres na perimenopausa e na pósmenopausa, não houve diferença estatística significativa pelo Teste Exato de Fisher (pvalor $=0,8138) . \quad$ Os resultados estão apresentados na figura 1.

Figura 1- Análise dos sintomas das mulheres no climatério, conforme o Índice de Kupperman e Bratt.

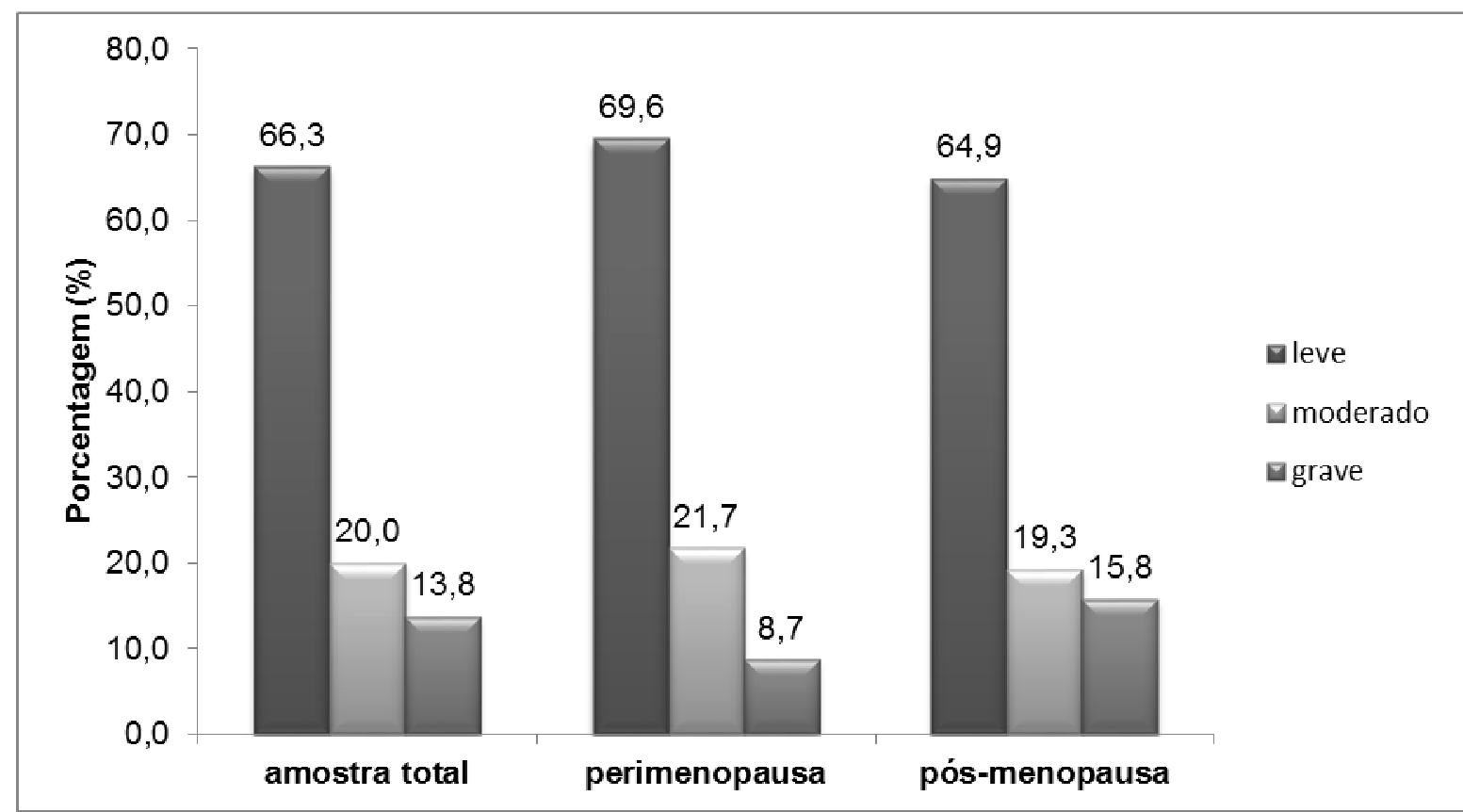

$\mathrm{Na}$ comparação da qualidade de verificou-se que não houve diferença vida de saúde da mulher nos estágios de significativa $\quad(\mathrm{p}$-valor $=0,7149)$. Os perimenopausa e de pós-menopausa, resultados estão demonstrados na Figura 2. 
Figura 2 - Qualidade de vida das mulheres na peri e pós-menopausa.

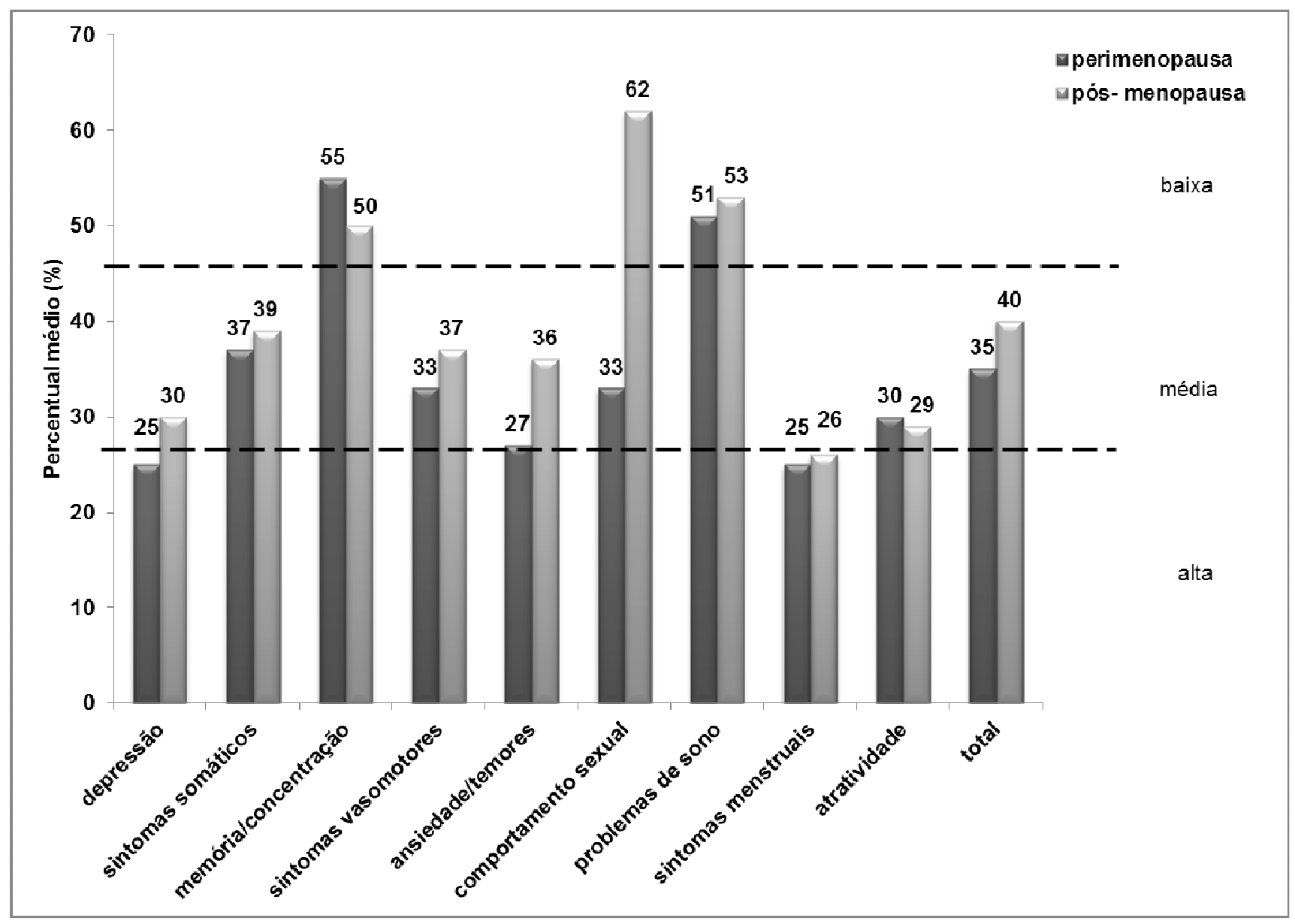

Também ao comparar o IMKB e a $\mathrm{QV}$, verificou-se que existe uma forte dependência entre a qualidade de vida e o índice IMKB (p-valor=0,00003). Enquanto as mulheres com índice leve têm qualidade de vida predominantemente alta, as que possuem índice moderado a grave, apresentam qualidade de vida média ou baixa.

Verificou-se que a $\mathrm{QV}$, em cada um dos seus domínios, independe do período menopausal, segundo o Teste Exato de Fisher (5\% significância). Os resultados estão demonstrados na Tabela 3. 
Tabela 3- Qualidade de vida da mulher segundo os domínios e o período menopausal, Alfenas, 2013-2014.

Domínios peri-menopausa pós-menopausa p-valor

Depressão

$\begin{array}{cccc}\text { Alta } & 12 & 31 & \\ \text { Média } & 3 & 16 & 0,1725 \\ \text { Baixa } & 8 & 10 & \end{array}$

Sintomas somáticos

$\begin{array}{rrrr}\text { Alta } & 11 & 28 & \\ \text { Média } & 1 & 9 & 0,3732 \\ \text { Baixa } & 11 & 20 & \end{array}$

Memória e concentração

$\begin{array}{crrr}\text { Alta } & 10 & 24 & \\ \text { Média } & 4 & 13 & 0,9014 \\ \text { Baixa } & 9 & 20 & \end{array}$

Sintomas vasomotores

$\begin{array}{crcc}\text { Alta } & 8 & 30 & \\ \text { Média } & 0 & 0 & 0,2162 \\ \text { Baixa } & 15 & 27 & \end{array}$

Ansiedade/ temores

$\begin{array}{rrrr}\text { Alta } & 15 & 34 & \\ \text { Média } & 0 & 0 & 0,8007 \\ \text { Baixa } & 8 & 23 & \end{array}$

\section{Comportamento sexual}

$\begin{array}{rrrr}\text { Alta } & 3 & 12 & \\ \text { Média } & 8 & 11 & 0,3468 \\ \text { Baixa } & 12 & 34 & \end{array}$

Sono

$\begin{array}{cccc}\text { Alta } & 3 & 9 & 1 \\ \text { Média } & 7 & 18 & 57 \\ & & \\ & \text { Revista da Universidade Vale do Rio Verde, Três Corações, v. 13, n. 1, p. 48-65, } 2015\end{array}$


$\begin{array}{lll}\text { Baixa } & 13 & 30\end{array}$

Sintomas menstruais

$\begin{array}{lll}\text { Alta } & 15 & 40\end{array}$

$\begin{array}{llll}\text { Média } & 0 & 0 & 0,7907\end{array}$

$\begin{array}{lll}\text { Baixa } & 8 & 17\end{array}$

\section{Atratividade}

$\begin{array}{lll}\text { Alta } & 11 & 21\end{array}$

$\begin{array}{lll}\text { Média } & 7 & 23\end{array}$

$\begin{array}{lll}\text { Baixa } & 5 & 13\end{array}$

No estudo, também foi realizada uma comparação estatística do grupo de mulheres que faziam uso de reposição hormonal $(\mathrm{n}=12)$ com as que não a faziam $(n=68)$. Na comparação dos sintomas do climatério, segundo o IMKB, não houve diferença estatística entre os dois grupos $(\mathrm{p}=0,9891)$.

\section{DISCUSSÃO}

O climatério pode ser interpretado como um processo de transformação físicoemocional fisiológico, não patológico, apesar de apresentar manifestações clínicas de acordo com a queda gradual dos hormônios e, principalmente, da individualidade da mulher. Assim, outros fatores podem agravar o estado físico e emocional dessas mulheres, tais como:
$\mathrm{Na}$ comparação dos grupos em relação à qualidade da saúde da mulher, também não houve diferença estatística significativa $\quad(p=0,6289) . \quad$ Mas, na comparação dos domínios desse mesmo instrumento, houve diferença apenas no domínio depressão ( $\mathrm{p}=0,0145)$, em que as mulheres com reposição hormonal apresentaram menos sintomas depressivos.

condições de vida; história reprodutiva; carga de trabalho; hábitos alimentares; tendência a infecções; dificuldade de acesso aos serviços de saúde para a obtenção de serviços e de informações, assim como outros conflitos socioeconômicos, culturais e espirituais associados ao período da vida e às individualidades (VALENÇA; NASCIMENTO FILHO; GERMANO, 2010). 
Nosso estudo optou por trabalhar com mulheres entre 40- 65, assim como outros autores (SILVA FILHO; COSTA, 2008; LORENZI et al., 2009a; ZAHAR et al., 2005) a fim de se contemplar as diversas fases do climatério.

As mulheres que participaram do estudo são em sua maioria branca, possuem companheiro fixo, apresentam alguma comorbidade e estão com sobrepeso ou são obesas. Esses parâmetros são também encontrados em outros estudos (SILVA FILHO; COSTA, 2008; MARTINS et al., 2009; LORENZI et al., 2009).

O sobrepeso e a obesidade encontrados na maioria da população deste estudo assim como os encontrados por outros autores (LORENZI et al., 2006; SILVEIRA et al., 2007) podem ser explicados pela deficiência hormonal, que pode levar ao aumento de peso, relacionado à redução do metabolismo basal, principalmente à gordura abdominal; também são devido à diminuição das atividades físicas regulares, associada com o aumento da ingestão calórica; e além disso, podem estar ligados à depressão (FERNANDES et al., 2008).

Dentre as comorbidades apresentadas, a hipertensão arterial sistêmica (HAS) foi prevalente no estudo, sendo também relatada entre as principais comorbidades encontradas por outros autores (FERNANDES et al., 2009; BADRAN et al.,2007 ). Estudo realizado por Fernandes al. (2008) relata que, eventualmente, $80 \%$ das mulheres apresentam HAS na menopausa. Isso pode estar relacionado ao aumento de peso, à redução da atividade física e da própria proteção hormonal ao sistema cardiovascular. Mas as VI Diretrizes Brasileiras de Hipertensão (DBHA V1, 2010) recomendam a terapia de reposição hormonal apenas para o tratamento de sintomas pós-menopausa, enfatizando que deverá ser prescrita na menor dose efetiva e durante curto período de tempo. A terapêutica de reposição hormonal não é recomendada para a prevenção primária ou secundária de doença cardiovascular, podendo mesmo aumentar o risco cardiovascular ou de outras doenças como câncer de mama, demência, tromboembolismo e colecistopatia. Assim, verificou-se durante o presente estudo que uma pequena parcela faz utilização da reposição hormonal, cujos médicos recomendam apenas quando verificam que não há riscos para a paciente e na presença de sintomas acentuados da menopausa.

$\begin{array}{cl}\mathrm{Na} & \text { análise dos } \\ \text { climatéricos, } & \text { por meio do IMKB, as }\end{array}$


mulheres apresentaram em sua maior proporção sintomas leves, tanto na amostra total quanto nos dois períodos do climatério. Assim, no presente estudo, verificou-se que as mulheres, mesmo as que não fazem reposição hormonal, se apresentam bem melhores do que os verificados em outros estudos (SILVA FILHO; COSTA, 2008; LORENZI et al., 2009), em que os sintomas encontrados foram de moderados a graves.

Em um estudo comparativo, realizado por Martins et al. (2009) com mulheres na pós-menopausa, usuárias e não usuárias de reposição hormonal, as primeiras apresentaram em média sintomas leves e as demais, moderados. Já em outros estudos (ZAHAR et al., 2005; GRAVENA et al., 2013) também comparativos entre usuárias e não usuárias de reposição hormonal, realizados com mulheres na pósmenopausa, em ambos os grupos a maioria dos sintomas foram leves.

Neste estudo, não se observou uma relação entre as fases da menopausa e a severidade dos sintomas climatéricos (IMKB; figura 1) e também no comprometimento dos domínios da qualidade de vida (figura 2). O que foi observado é a influência da severidade dos sintomas climatéricos na qualidade de vida. A mesma constatação foi verificada em estudos de SANTOS et al., (2007), realizado com mulheres nas Unidades da Estratégias de Saúde da Família, localizadas na zona leste do município de São Paulo.

Buscou-se determinar o perfil da saúde da mulher climatérica com base no impacto que os sintomas da menopausa exerceram sobre a qualidade de vida. $\mathrm{Na}$ análise, em relação aos percentis, em que a pontuação de zero a 27 corresponde à melhor qualidade de vida, as mulheres na fase de perimenopausa apresentaram-se melhores em relação à depressão, à ansiedade/temores e aos sintomas menstruais. Já na pós-menopausa só apresentaram em relação aos problemas menstruais. Quanto ao comprometimento da QV, em relação aos percentis (pontuação acima de 46), as mulheres na perimenopausa apresentaram maior comprometimento nos domínios concentração/memória, em problemas de sono e em sintomas somáticos, enquanto as mulheres na pós-menopausa apresentaram nos domínios comportamento sexual, problemas de sono e concentração/memória.

O comprometimento da QV com relação ao comportamento sexual nas mulheres na pós-menopausa pode estar relacionado com as próprias alterações 
advindas da menopausa, como a secura vaginal e a diminuição da libido (GARCIAVINIEGRAS; PORTA, 2003). Além disso, a associação entre sintomas do climatério e alguns tipos de medicamentos antidepressivos pode estar relacionada com as alterações na função sexual.

Em um estudo de Oliveira; Jesus; Merighi (2008), realizado em Juiz de Fora (MG), com participantes de um grupo de climatério de uma unidade de saúde da família, verificou-se no relato das entrevistadas a diminuição da libido e a perda do prazer na relação sexual, a presença da secura vaginal e até mesmo uma repulsa ao companheiro. Em outro estudo (LORENZI et al., 2006), realizado em Caxias do Sul, observou-se que não somente os fatores biológicos, mas também os culturais e os psicossociais tendem a comprometer a qualidade de vida no climatério.

Assim, o climatério abrange não apenas os sintomas decorrentes da deficiência estrogênica, mas, acima de tudo, um contexto amplo que contempla a inserção social, no qual se mantêm interrelações de diversas naturezas, para que se possa usufruir de todo o potencial criativo e construtor. Dessa forma, a percepção dos sintomas e dos sentimentos desencadeados pelo climatério determinará a qualidade de vida (LINDH-ÅSTRAND et al., 2007).

Diversos estudos (VALENÇA; NASCIMENTO FILHO; GERMANO, 2010; FREITAS, SILVA; SILVA, 2004; ZAMPIERI et al., 2009) demonstram que as mulheres apresentam falta de conhecimento acerca do climatério. Muitas mulheres desconhecem o termo, outras atribuem como sinônimo de menopausa e ainda há as que não sabem nem mesmo o que é a menopausa. O desconhecimento gera incertezas sobre o climatério e cria mitos, fazendo com que essa fase da vida seja mais conturbada e colabore ainda mais para o comprometimento da qualidade de vida.

Existe um consenso entre alguns autores (VALENÇA; NASCIMENTO FILHO; GERMANO, 2010; FERNANDES et al., 2008; SILVEIRA et al., 2007; FREITAS; SILVA; SILVA, 2004) da necessidade de atendimento mais humanizado e holístico, que possibilite a essas mulheres entender essa fase de sua vida, expor suas dúvidas e medos. Os profissionais de saúde precisam compreender as necessidades das mulheres nesse período para que possam orientá-las de forma a atender suas necessidades e a estimular suas potencialidades. 
Um atendimento humanizado, com uma escuta qualificada, bem como a criação de grupos de climatério, faz-se necessário nas unidades de saúde. Assim, a equipe multiprofissional poderá atender a essas mulheres de forma integral e, assim, estas poderão compartilhar suas experiências, seus medos e suas expectativas.

\section{CONCLUSÕES}

Neste estudo, a maioria das mulheres no climatério apresentaram

\section{REFERÊNCIAS}

\section{Associação Brasileira de Climatério -}

SOBRAC. Terapêutica hormonal na peri e na pós-menopausa. Consenso da SOBRAC 2004; p.5-39.

2.Badran, A.V.; Araújo, A.L.L.; Nagae, D.K.I.; Takahashi, L.R.; Formícola, N.R.; Miyamoto, W.R. et al. Aspectos da sexualidade na menopausa. Arq. Med.

Hosp. Fac. Cienc. Med., v.52, n.22, p.3943, 2007.

3.Bonganha, V.; Madruga, V.A. Qualidade de Vida da Mulher na Pós-Menopausa. In: VILARTA R, GUTIERREZ GL, sintomas leves e a qualidade de vida mostrou-se influencida pelos sintomas identificados. Verificou-se que a presença dos sintomas leva as mulheres a procurar por unidades de saúde. Faz-se necessário que estas unidades estejam preparadas para um atendimento humanizido e holístico a fim de que as mulheres sejam atendidas de forma integral e tenham suas necessidades sanadas, proporcionando assim uma melhora na qualidade de vida.

\section{MONTEIRO IN (organizadores).}

Qualidade de vida: evolução dos conceitos e práticas no século XXI. Campinas: Ipes, cap. 4, p. 37-43, 2010.

4.BRASIL. Ministério da Saúde. Secretaria de Atenção à Saúde. Departamento de Ações Programáticas Estratégicas. Manual de Atenção à Mulher no Climatério/Menopausa. - Brasília: Editora do Ministério da Saúde, 2008.

\section{Carvalho, J.A.M.; Garcia, R.A. O}

envelhecimento da população brasileira: 
um enfoque demográfico. Cad. Saúd.

Públ., v.19, n.3, p.725-33, 2003.

6.Dias, R.S.; Ramos, C.C.; Kerr, C.F.;

Trinca, L.A.; Cerqueira, A.T.A.R.; Dalben,

I. et al. Adaptação para o português do questionário de autoavaliação de percepção de saúde física e mental da mulher de meiaidade - Questionário da Saúde da Mulher.

Rev. Psiq. Clín., v.29, n.4, p. 181-89, 2002.

7.Fernandes, C.E. (coordenador). Consenso Brasileiro Multidisciplinar de Assistência à Saúde da Mulher Climatérica. São Paulo: SOBRAC; Rio de Janeiro: FEBRASGO, 2003.

8.Fernandes, C.E.; Pinho-Neto, J.S.L.; Gebara, O.C.E.; Santos Filho, R.D.; Pinto Neto, A.M.; Pereira Filho, A.S. et al. I Diretriz Brasileira sobre Prevenção de doenças cardiovasculares em mulheres climatéricas e a influência da terapia de reposição hormonal (TRH) da Sociedade Brasileira de Cardiologia (SBC) e da Associação Brasileira do Climatério (SOBRAC). Arq. Bras. Cardiol., v. 91, n.1( supl.1), p.1-23, 2008.

9.Fernandes, R.C.L.; Silva, K.S.; Bonan, C.; Zahar, S.E.V.; Marinheiro, L.P.F.
Avaliação da cognição de mulheres no climatério com o Mini-Exame do Estado Mental e o Teste de Memória da Lista de Palavras. Cad. Saúde Públ., v.25, n.9, p.1883-93, 2009.

10.Freitas, K.M.; Silva, A.R.V.; Silva, R.M. Mulheres vivenciando o climatério. Acta Sci. Health Sci., v.26, n.1, p. 121-28, 2004.

11.Gallon ,C.W.; Wender, M.C.O. Estado nutricional e qualidade de vida da mulher climatérica. Rev. Bras. Ginecol. Obstet., v. 34, n.4, p. 175-83, 2012.

12.Garcia-Viniegras, C.R.V.; Porta, S. M. Climaterio y bienestar psicológico. Rev.

Cubana Obstet. Ginecol., v. 29, n.3, 2003.

13.Gravena, A.A.F.; Rocha, S.C.; Romeiro, T.C.; Agnolo, C.M.D.; Gil, L.M.; Carvalho, M.D.B. et al . Sintomas climatéricos e estado nutricional de mulheres na pósmenopausa usuárias e não usuárias de terapia hormonal. Rev. Bras. Ginecol.

Obstet., v. 35, n.4, p. 178-84, 2013.

14.Kupperman, H.S.; Blatt, M.H.G. Menopausal indice. J. Clin. Endocrinol., v.15, n.1, p. 688-694, 1953. 
15.Lindh-Åstrand, L.; Hoffmann, M.;

Hammar, M.; Kjellgren, K.I. Women’s conception of the menopausal transition a qualitative study. J. Clin. Nurs., v.16, n.3, p.509-17, 2007.

16.Lorenzi, D.R.S.; Baracat, E.C.; Saciloto, B.; Padilha Jr. I. Fatores associados à qualidade de vida após menopausa. Rev.

Assoc. Med. Bras., v.52, n.5, p.312-17, 2006.

17.Lorenzi, D.R.S.; Catan, L.B.; Cusin, T.;

Felini, R.; Bassani, F.; Arpini, A.C. Caracterização da qualidade de vida segundo o estado menopausal entre mulheres da Região Sul do Brasil. Rev. Bras. Saúde Mater. Infant., v.9, n.4, p. 459-66, 2009.

18.Martins, M.A.D.; Nahas, E.A.P.; NahasNeto, J.; Uemura, G.; Buttros, D.A.B.; Traiman, P. Qualidade de vida em mulheres na pós-menopausa, usuárias e não usuárias de terapia hormonal. Rev. Bras. Ginecol.

Obstet., v.31, n.4, p. 196-202, 2009.

19.Moraes, V.M.; Vandenberghe, L.; Silveira, N.A. Humor, atenção concentrada e qualidade de vida no climatério: um estudo no Brasil Central. Psic., Saúde \& Doenças, v.8, n.2, 2007.
20.Oliveira, D.M.; Jesus, M.C.P.; Merighi, M.A. Climatério e sexualidade: a compreensão dessa interface por mulheres assistidas em grupo. Texto \& Contexto, v.17, n.3, p.519-26, 2008.

21.Santos, L.M.; Eserian, P.V.; Rachid, L.P.; Cacciatore, A.; Bourget, I.M.M.; Rojas, A.C. et al. Síndrome do climatério e qualidade de vida: uma percepção das mulheres nessa fase da vida. Rev. APS, v.10, n.1, p.20-6, 2007.

22.Silva Filho, E. A.; Costa, A.M. Avaliação da qualidade de vida de mulheres no climatério atendidas em hospital-escola na cidade do Recife, Brasil. Rev. Bras. Ginecol. Obstet., v.30, n.3, p. 113-20, 2008.

23.Silveira, I.L.; Petronilo, P.A.; Souza, M.O.; Silva, T.D.N.C.; Duarte, J.M.B.P.; Maranhão, T.M.O. et al . Prevalência de sintomas do climatério em mulheres dos meios rural e urbano no Rio Grande do Norte, Brasil. Rev. Bras. Ginecol. Obstet., v.29, n.8, p.415-22, 2007.

24. Soares, G.R.S.; Simões, S.M.F.; Fazoli, K.L.S.; Coutinho, F.H.; Cortez, E.A. El vivir de las mujeres en el climaterio: revisión sistemática de la literatura. 
Enferm. Global, v.11, n.25, p.440-50,

2012.

25.Sociedade Brasileira de Cardiologia. VI

Diretrizes Brasileiras de Hipertensão. Arq.

Bras. Cardiol., v. 95, n.1 (supl.1), p.1-5,

2010.

26.Valença, C.N.; Nascimento Filho, J.M.;

Germano, R.M. Mulher no climatério:

reflexões sobre desejo sexual, beleza e

feminilidade. Saúde Soc., v.19, n.2, p. 273-

85, 2010.

27.Zahar, S.E.V.; Aldrighi, J.M.; Pinto

Neto, A.M.; Conde, D.M.; Zahar, L.O.;

Russomano, F. Qualidade de vida em

usuárias e não usuárias de terapia de

reposição hormonal. Rev. Assoc. Med.

Bras., v. 51, n.3, p.133-8, 2005.

28.Zampieri, M.F.M.; Tavares, C.M.A.;

Hames, M.L.C.; Falcon, G.S.; Silva, A.L.;

Gonçalves, L.T. O processo de viver e ser

saudável das mulheres no climatério. Rev.

Enferm., v.13, n.2, p. 305-12, 2009.

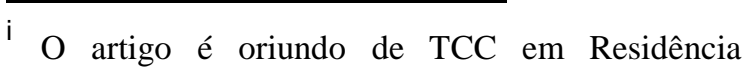
Multiprofissional em Saúde da Família.

Autor correspondente: Walnéia Aparecida de

Souza - Universidade Federal de Alfenas-MG

Rua Gabriel Monteiro da Silva, 700 - Centro

Alfenas- MG

Telefone: 35 - 32991341 